\title{
Neuroendoscopy: What an Anaesthesiologist Should Know?
}

\section{Hemlata* \\ Department of Anaesthesiology, King George's Medical University, India}

*Corresponding author: Hemlata, Department of Anaesthesiology, King George's

Medical University, Lucknow, UP, India, Tel: +91-9415410236; Email: hema2211@yahoo.co.in

\section{Review Article}

Volume 4 Issue 3

Received Date: July 15, 2019

Published Date: August 06, 2019

DOI: $10.23880 /$ accmj-16000157

\section{Abstract}

Although neuroendoscopy has become popular both as an independent treatment modality and as an adjunct to micro-neurosurgery for various neurologic disorders, yet we have very sparse literature about basic anaesthesia concepts for this particular technique. Anaesthesiologists can positively contribute to overall neurologic outcome of these procedures through attention to the medical condition of the patient, the basic surgical requirements, specific anaesthetic requirements, and heightened awareness of specific intraoperative and postoperative risks. General anaesthesia with endotracheal intubation remains the technique of choice. Though this is a minimally invasive procedure, invasive haemodynamic monitoring should be done as there is high incidence of haemodynamic instabilities during these procedures. Direct or indirect measurement of ICP is crucial to identify sudden and dangerous increases in ICP. Bradycardia is the commonest arrhythmia seen intraoperatively and generally responds to simple manoeuvres like removal of scope, decreasing the speed of inflow of irrigation fluid and allowing its egress. Use of atropine and other resuscitative measures are needed rarely. Warmed lactated ringer solution can be safely used for intraoperative irrigation with minimal postoperative impact. Close observation of vital signs, serum electrolytes as well as volume and temperature of the irrigation fluid and close communication between anesthesiologist and surgeon, are prerequisites for better outcome. Close postoperative monitoring is required to diagnose and treat complications such as convulsions, persistent hydrocephalus, blocked stoma, CSF leak, haemorrhage, post-operative intracranial haematomas, diabetes insipidus, electrolyte imbalance, infections etc. thus improving overall outcome.

Keywords: Neuroendoscopy; Endoscopic third ventriculostomy; Anaesthesia; Intracranial pressure; Cerebral perfusion pressure; Bradycardia; Arrhythmia

Abbreviations: ETV: Endoscopic Third Ventriculostomy; NS: Normal Saline; RL: Lactated Ringer's Solution; VAE: Venous Air Embolism; TCD: Transcranial Doppler; MB: Mammillary Body.

\section{Introduction}

Neuroendoscopic procedures are becoming more frequent and popular in the treatment of intracranial 


\section{Anaesthesia \& Critical Care Medicine Journal}

disease. This technique allows intracranial intervention with minimal damage to healthy brain tissue. It also provides surgeons better visualization of the intracranial structures. Initially, neuroendoscopy was performed exclusively for endoscopic third ventriculostomy (ETV), for the treatment of obstructive hydrocephalus. However, in recent times, it is being used successfully for many types of neurosurgical procedures, either as a primary approach or as an adjunct [1].

Although a minimally invasive technique, with low morbidity and mortality even in paediatric patients, a few complications have been reported in the literature [2]. Although many of the neuroendoscopic procedures have become established as a routine for the last 10-20 years, yet we have very sparse literature about basic anaesthesia concepts for this particular technique [3]. For successful conduct of anaesthesia, it is important for anaesthesiologist to be aware of the associated complications as well as the basic surgical requirements and specific anaesthetic requirements.

\section{History}

Neuroendoscopy was first introduced by a Chicago urologist, Victor Darwin Lespinasse in the year 1910 [4]. In an attempt to decrease cerebrospinal fluid (CSF) production, he penetrated the lateral ventricles of two hydrocephalic children with the rigid cytoscope and fulgurated the choroid plexus. Later-on, in 1922, Dandy coined the term ventriculoscope and described the use of a rigid Kelly cytoscope to inspect the floor of the lateral ventricles in patients with hydrocephalus [5]. In 1923, Mixter performed the first endoscopic ventriculostomy in a child with congenital obstructive hydrocephalus [6]. However, neuroendoscopic procedures did not gain wide acceptance and their popularity declined in the subsequent years due to equipment limitations and high surgical morbidity and mortality. The birth of microneurosurgery in the 1960s pushed endoscopy further into the background. With technological advances in the optics and miniaturization, neuroendoscopic procedures have once again become very popular in the recent years [7-9].

\section{Brief Description of the Procedure}

Neuroendoscopy is a surgical technique which uses small thin endoscopes (around $5 \mathrm{~mm}$ in diameter) to approach deep structures of the brain and perform surgical procedures there. There are three basic prerequisities of doing neuroendoscopy:
- Preformed space into which the endoscope can be passed

- A sufficient light has to be delivered into the cavity to visualise the structures inside

- We must be able to pass instruments into that space to perform surgical procedures

The technique employs burr-hole access to the cranium with placement of a rigid or flexible scope through the frontal cortex into the ventricle (Figure 1). Adequate visualization requires continuous irrigation of the ventricles with warmed normal saline (NS) or lactated Ringer's solution (RL) accompanied by drainage of CSF and irrigating fluid through the scope or the bur-hole. Patient selection criteria are important to ensure a successful neuroendoscopy.

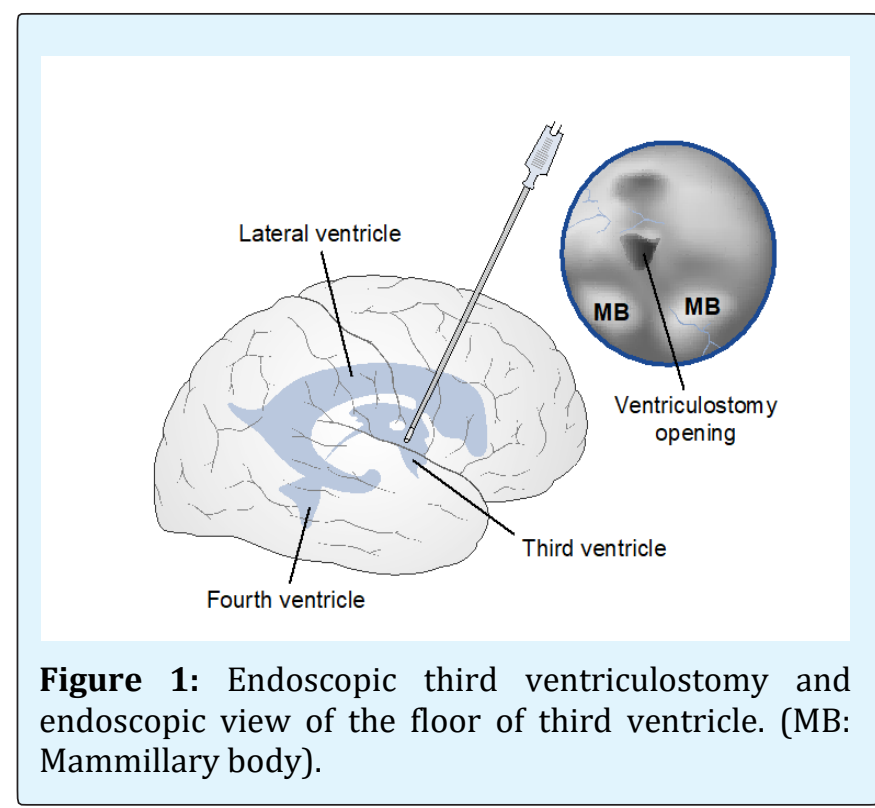

\section{Indications}

This procedure has been successfully used for various diagnostic as well as therapeutic applications in a variety of intracranial procedures (Table 1). Its most prominent application is in the treatment of noncommunicating hydrocephalus via ETV [10]. The surgeon establishes a connection between the third ventricle and the subtentorial subarachnoid space by endoscopically fenestrating the floor of the third ventricle (Figure 1). The overall success rate of ETV is $60 \%$ to $90 \%$ with a surgical risk of approximately 5\% for significant morbidity [1113]. 


\section{Anaesthesia \& Critical Care Medicine Journal}

\begin{tabular}{|c|c|c|}
\hline \multicolumn{2}{|c|}{ Pure Endoscopic Procedure } & Endoscopic Assisted Microsurgery \\
\hline Diagnostic & Therapeutic & \\
\hline $\begin{array}{l}\text { Ventriculoscopy } \\
\text { Biopsy of intracranial } \\
\text { tumors }\end{array}$ & $\begin{array}{c}\text { Endoscopic third ventriculostomy } \\
\text { Evacuation of intracranial hematoma } \\
\text { Drainage of cystic tumors } \\
\text { Removal of intra/paraventricular } \\
\text { tumor and nontumoral lesions } \\
\text { Evacuation of brain abscesses } \\
\text { Fenestration of loculated intraventricular cysts, } \\
\text { occluded Foramen of Monroe } \\
\text { Fulgaration of choroid plexus } \\
\text { Retrieval of displaced shunt or foreign bodies } \\
\text { Aneurysm surgery }\end{array}$ & $\begin{array}{c}\text { Pituitary tumor resection } \\
\text { Skull base tumor biopsy } \\
\text { Cerebral aneurysms } \\
\text { Microvascular decompression } \\
\text { Acoustic neuromas }\end{array}$ \\
\hline
\end{tabular}

Table 1: Indications of Neuroendoscopy.

Neuroendoscopy is also used for fenestration of loculated intraventricular cysts, trapped ventricular cavities and occluded foramen of Monro [14,15]. Other uses include biopsies or complete removal of intraventricular and periventricular tumors and nontumoral lesions like neurocysticercosis [16]; drainage of cystic tumors under direct vision; intraventricular fulgaration/cauterization of choroid plexus [17]; evacuation of intracerebral hematoma and brain abscesses, and endocavitory syringostomy [18]; retrieval of displaced shunts and other foreign bodies [19]. Besides this, endoscopic assistance is required in many microsurgeries.

\section{Preoperative Concerns}

A thorough preanaesthetic assessment of the patient should be carried out. Children with hydrocephalus may have other associated multisystem congenital defects. Patients may present with symptoms of raised ICP, like vomiting, headache, confusion or obtundation. Any electrolyte abnormality or significant dehydration as a result of prolonged or recurrent nausea and vomiting needs to be corrected prior to surgery. Patient's detailed neurologic examination should be done preoperatively and documented. Since the procedure is usually of short duration, so premedication with anxiolytics or narcotics should be titrated very carefully to avoid oversedation which may cause hypoventilation and hypercarbia in the postoperative period. Long acting benzodiazepines are often avoided. Preoperative sedatives are best avoided in patients with depressed consciousness. Margetis, et al. used intravenous corticosteroids preoperatively to reduce the potential risk of chemical ventriculitis and subsequent hydrocephalus that may occur due to intraventricular spillage of colloid material during endoscopic resection [20].

\section{Intraoperative Concerns}

The primary anaesthetic goals of any neuroendoscopic procedure include:

- Intraoperative immobilization (head is frequently fixed in a head frame)

- Haemodynamic stability

- Prevention, detection and treatment of sharp increases in ICP (this requires good communication with the neurosurgeon)

- Rapid emergence to allow early neurologic examination.

All the above anaesthetic goals dictate our choice of anaesthesia as well as irrigating solutions and intraoperative monitoring. Even though there is minimal brain penetration associated with neuroendoscopy, there can be significant intraoperative events owing to intracranial circulatory insufficiency due to the pressure generated by the continuous irrigation system [21].

\section{Choice of Anaesthetic Technique}

A number of different anaesthetic regimens have been used for these procedures depending on the experience and practice setting of the anaesthesiologist. Though the procedure can be conducted under sedation and local anaesthesia, but general anaesthesia is mostly required along with endotracheal intubation to ensure patient immobility, a secured airway and controlled ventilation. Intravenous propofol or thiopental sodium can be used 


\section{Anaesthesia \& Critical Care Medicine Journal}

for induction of anaesthesia. Alternatively, inhalational anaesthetics such as sevoflurane or halothane can be used in small children. Desflurane is known to cause respiratory tract irritation, coughing, laryngospasm, excitatory phenomenon and cardiovascular stimulation and thus may increase the ICP [22]. Hence, it is not preferred for the induction of anaesthesia. However, it can be safely used during the maintenance of anaesthesia and allows for quicker emergence on its termination $[22,23]$. Derbent, et al. suggested use of sevoflurane with $\mathrm{ETCO}_{2}$ at $30 \pm 2 \mathrm{mmHg}$ to reduce its cerebral vasodilatory effects [24]. They avoided remifentanil to maintain normotension. Baykan, et al. found no difference in using either alfentanil or remifentanil as the opioid of choice in their technique [3].

Nondepolarizing neuromuscular blocking agents are used to facilitate endotracheal intubation and for maintenance of muscle relaxation [21,25-27]. Succinylcholine may be used if a difficult airway is anticipated. Intravenous lidocaine $(1.5-2 \mathrm{mg} / \mathrm{kg})$ may be used to attenuate elevation of ICP in response to laryngoscopy and intubation. Maintenance is done using inhalational anaesthetic and 50\% oxygen in air with controlled ventilation of lungs. Nitrous oxide is generally avoided to prevent elevations in ICP as it may diffuse into air trapped in the ventricles and subdural space following decompression of the ventricles [24]. Some studies recommend that $\mathrm{N}_{2} \mathrm{O}$ should be stopped after encountering single episode of venous air embolism (VAE) [28].

Derbent, et al had suggested that their anaesthetic technique together with the use of $0.9 \% \mathrm{NaCl}$ for intravenous fluid replacement produced a lower incidence of adverse haemodynamic events [24]. Short acting narcotics such as fentanyl or alfentanyl are preferred analgesic agents. Morphine and meperidine should be avoided as they have active metabolites. Hyperventilation can be employed to maintain mild to moderate hypocarbia till the time ventricles are decompressed. Maintenance of an euvolemic state should be our main aim for intraoperative fluid management.

\section{Irrigation solutions}

The selection and volume of irrigating fluid can affect the CSF composition and might cause an increase in postoperative morbidity [29]. Though the use of normal saline(NS) at body-temperature as irrigating fluid has been advocated, but large volume irrigation of $\mathrm{NS}(\geq 500$ $\mathrm{mL}$ ) may cause CSF acidosis [29,30]. Salvador et al found a significant correlation between changes in CSF composition and the total volume of irrigation solution used, but no correlation with the duration of neuronavigation. A cut-off point of $500 \mathrm{ml}$ of saline irrigation solution has been recommended. Warmed (at body temperature) lactated Ringer solution (RL) is the most frequently used irrigation fluid $[3,25,31]$ and is being used preferably at our centre as well.s: RL has been found to result in postoperative hyperkalemia in some patients [32]. To avoid intraoperative and postoperative complications arising from the use of irrigating fluids, care should be taken to limit the loss of CSF and to use irrigation only when necessary [33].

\section{Intraoperative Monitoring}

Besides standard anaesthesia monitoring (electrocardiography, noninvasive blood pressure, pulse oximetry, end-tidal carbon dioxide, and body temperature), direct intra-arterial pressure and beat-tobeat HR monitoring should be done as there is high incidence of haemodynamic instabilities during these procedures $[25,26,29,31]$. that patient monitoring for ETV should be just like any major surgery and not like a minimally invasive surgery [3]. Whenever central line placement is required, care should be taken to avoid unintended puncture of the peripheral shunt tubing. Inadequate venting of the irrigation solution can cause precipitous decrease in CPP [34]. Therefore, continuous measurement of CPP should be done $[26,35]$. The pressure inside the neuroendoscope (PIN) can be measured easily and maybe helpful as it correlates well with ICP and CPP $[21,36]$. Study by Salvador demonstrated that the PIN can be a reliable surrogate measure of ICP [37].

To measure PIN, a fluid-filled catheter connected to a stop-cock, is connected to the irrigation lumen of the neuroendoscope and attached to a pressure transducer zeroed at the skull base [38]. It has been recommended that intraoperative CPP should be maintained above $40 \mathrm{mmHg}$ at all times [26]. Kalmar, et al. observed that the Cushing reflex developed in almost every case when CPP dropped below $15 \mathrm{mmHg}$; however, the occurrence of bradycardia was not always associated with a low CPP. They further found that the occurrence of hypertension and tachycardia ("atypical Cushing reflex") was the result of an increase in ICP [26]. Transcranial Doppler (TCD) can be used to measure middle cerebral artery blood flow velocity through the temporal window. However, it is difficult to maintain a reliable TCD signal at all times during the surgery 


\section{Anaesthesia \& Critical Care Medicine Journal}

\section{Intraoperative Complications}

The incidence of intraoperative and postoperative complications of neuroendoscopy range widely from $5 \%$ to $30 \%[36,39]$. However, operative mortality has been reported to be very low (0\%-1\%). A good communication between the surgeon and the anaesthesiologist is very important to ensure prompt intervention in case of any complications as it may be difficult for the anaesthesiologist to follow the progress of the procedure. Besides other intraoperative monitors, the colour and the volume of the returning perfusate should be observed during the procedure to have a fair idea about the degree of blood loss. The intraoperative complications of neuroendoscopy include haemodynamic disturbances, arrhythmias, intraventricular haemorrhage, hypothermia, pneumocephalus/pneumoventricle, fornix injury, hypothalamic damage, cranial nerve damage, visual obscuration, failed ETV etc. (Table 2) Most of the complications occur because of sudden CSF drainage or because of irrigant fluid or instrumentation. More recently, nasal endoscopic approach has been used to perform skull base surgery for aneurysms and tumours. With transnasal approach, severe intraoperative haemorrhage is the most important complication to consider followed by direct injury to surrounding neural structures.

\begin{tabular}{|c|c|}
\hline Intraoperative Complications & Post-operative Complications \\
\hline Intraventricular bleeding & \\
Pneumocephalus/pneumoventricle & Early postoperative complications \\
Bradycardia & Delayed awakening \\
Hypothermia & Blocked stoma \\
Fornix injury & Subdural hematoma \\
Hypothalamic damage & Intra-cerebral hemorrhage \\
Cranial nerve damage & CSF leak \\
Visual obscuration & Late complications \\
Failed ETV & \\
\hline
\end{tabular}

Table 2: Intaoperative and Postoperative Complications of ETV.

\section{Haemodynamic changes}

Sudden change in intracranial pressure due to CSF drainage or excessive use of irrigation fluid can lead to wide range of haemodynamic effects ranging from minor changes in heart rate and blood pressure to that of near fatal cardiac arrest $[40,41]$. Cardiac arrhythmias are common and range from bradycardia, tachycardia, premature ventricular contractions, supraventricular tachycardia to asystole. The proposed mechanisms include hypothalamic stimulation or damage [3,32,41-43] and acute rise in ICP [21,25,26]. Reduced regional cerebral perfusion during fenestration of third ventricle may result in ischaemia of the hypothalamic centres and medulla oblongata [3].

During ETV, the floor of the third ventricle is usually perforated in front of mamillary bodies located on the posterior hypothalamus. It is proposed that the traction exerted on this area during perforation inhibits the sympathetic activity and results in bradycardia [32]. Stimulation of posterior hypothalamus causes increased HR and BP, whereas stimulation of preoptic area causes decreased HR and BP [32]. Alfrey et al. also speculated that sudden ventricular decompression and intracranial hypotension causes rostral migration of the brain resulting in arrhythmias [42]. Fortunately, these haemodynamic changes are often transient and generally respond to simple manoeuvres like removal of scope, decreasing the speed of inflow of irrigation fluid and allowing its egress [37,44]. Use of atropine and other resuscitative measures are needed rarely [3].

Proano et al. reported significant bradycardia during the perforation of the third ventricle [43]. Baykan, et al. reported $26.8 \%$ incidence of bradycardia during balloon dilatation of the third ventricle [3]. Simply deflating the balloon resolved bradycardia in most of the patients; atropine being required only in 3 out of 59 cases $(1.41 \%$ of total). In a study by Ganjoo, et al. $10 \%$ of patients developed hypertension associated either with bradycardia or tachycardia but never manifested hypertension alone [44]. In contrast to these studies, Derbent, et al. did not report any haemodynamic changes during the procedure except for bradycardia in one patient which occurred during fenestration of the floor of the third ventricle [24]. Occurrence of bradycardia during ETV can be prevented by judicious use of irrigation at a speed of $\leq 10 \mathrm{ml} / \mathrm{min}$ and avoiding local pressures on the underlying hypothalamus [45]. Very high ICPs up to 150 


\section{Anaesthesia \& Critical Care Medicine Journal}

mmHg can quickly occur during neuroendoscopy, if the inflow of irrigating fluid is too rapid $[21,36]$ or if the outflow line is accidentally occluded [31], and this may remain unnoticed if ICP is not monitored continuously $[21,25,26,28,31,36]$.

\section{Haemorrhage}

Massive intraventricular bleeding due to damage to the ependymal vessels or the basilar artery lying beneath the floor of the third ventricle has been reported by different authors. Causes include excessive side movements of the scope, wrong entry in the lateral ventricle, removing flexible scope in curved tip position, repeated introduction of endoscope without the use of the peel-away sheath or other sheath [45]. Rarely blood might trickle from the burr-hole site into the ventricle, hence surgeons are advised to achieve proper haemostasis before entering the ventricle. Knowledge of the position of basilar artery and its branches and perforation of the floor anterior to these vessels help in avoiding injury.

Small haemorrhages during the procedures are usually venous in origin. It is usually self-limited or can be controlled using copious irrigation with warm RL solution or by inflating the balloon catheter. Cauterization may be attempted if a bleeding site can be identified. Intermittent careful closure of outflow channel can also produce tamponade effect. Anaesthesiologist should be vigilant enough to keep track of the blood loss and should remain prepared to transfuse blood components as and when required.

Once bleeding is stopped, intra-ventricular blood should be evacuated and sheath removed. A ventricular drain should be placed in case of residual oozing. Fluid should be replaced with air to prevent ventricular collapse and its consequences. When there is significant bleeding not controlled by any other technique, rapid conversion to open surgery is recommended.

\section{Hypothermia}

Significant hypothermia may occur during neuroendoscopy especially in small children, and can result in delayed awakening and disordered coagulation. Causes include large exchanges of irrigation fluid and ventricular CSF, wetting of drapes by the returning fluid, low environment temperature and impaired temperature regulation due to hypothalamic injury. Pre-warmed irrigation fluid and blankets should be used routinely to protect against intraoperative hypothermia [28,46].
Drainage line should be connected to outflow channel to avoid wetting of drapes.

\section{Pneumocephalus}

Excessive loss of CSF, improper site of burr-hole and use of nitrous oxide anaesthesia can all predispose to the development of pneumocephalus, which can interfere with direct visualization of the anatomic landmarks thus compromising the safety of the procedure. Nitrous oxide should either be avoided completely or it should be stopped after encountering even a single episode of VAE $[24,28]$.

\section{Hypothalamic damage}

Hypothalamic damage is a common complication of ETV. Although blunt technique for perforation of ventricular floor is better to avoid vascular damage, it may cause excessive traction on the hypothalamus in thick third ventricular floor. Hypothalamic damage can be avoided by perforation in the midline, between infundibular recess and mammillary body, and use of sharp technique. Anaesthesiologist should be aware of possibility of hypothalamic damage and its consequences like diabetes insipidus, loss of thirst, hyperphagia, amenorrhea, varying degree of drowsiness, hyperkalemia, hypernatremia, hypothermia and death [45].

\section{Fornix injury}

Fornix injury is one of the most common complications of intra-ventricular endoscopy and surgeon should take adequate measures to prevent it. It can be prevented by proper planning of burr-hole, avoiding significant side movements, selecting proper cases with significantly enlarged foramen of Monro and third ventricle, use of small size scope, limiting brain penetration to less than $5-6 \mathrm{~cm}$ from burr-hole, enlargement of foramen of Monro by hydrodissection and shrinkage of choroid plexus at the foramen by bipolar coagulation [47]. Flexible scope should be in the neutral position at the time of removal to avoid injury.

\section{Cranial Nerve Damage}

Injury to oculomotor and abducens nerve can occur when floor is bulging downward and the perforation is not made in the midline. Introducing the perforating instruments blindly far below the floor can also cause injury to cranial nerves [45]. Burr-hole should be placed as medially as possible and the perforations should be made in the midline. Surgeon should appreciate anatomy 


\section{Anaesthesia \& Critical Care Medicine Journal}

and should perforate the tough membrane using sharp instruments.

\section{Failed ETV}

At times, it may be difficult to perform ETV due to various anatomical and technical factors. Technical failure should be considered a complication because, even if the patient suffers no direct consequences from the procedure, he or she is still exposed to the risks of avoidable anaesthesia and an unnecessary surgical procedure. Proper pre-operative knowledge of the anatomical structures and careful selection of cases can reduce the risk of failed ETV [48].

\section{Postoperative \\ Complications}

Concerns

and

Close postoperative monitoring of vital signs, level of consciousness, and change in pupillary size should be done in all the patients who have undergone neuroendoscopy to detect any intracranial complications. Common problems in the immediate postoperative period include delayed awakening, confusion, hyperkalemia, transient pupillary dysfunction, transient hemiplegia, and memory loss $[32,36,39]$. Transient neurologic deficits are the most common postoperative complication, occurring in $8 \%$ to $38 \%$ of patients [36,39]. Delayed awakening is a major concern to the anesthesiologist. High levels of PIN are reportedly associated with delayed awakening and a higher rate of postoperative complications [36]. Salvador et al. found a strong correlation between increased PIN over $30 \mathrm{mmHg}$ and increases in postoperative complications [49]. Careful control of irrigating pressure therefore may reduce postoperative risk and avoid delayed emergence. Enya et al stressed the need for apnoea monitoring in the post-operative period as respiratory arrests have been reported in infants during the first hours after neuroendoscopy [50].

Other early post-operative complications include infections, fever, blocked stoma, CSF leak and post-operative intracranial hematomas. Diabetes insipidus, weight gain, precocious puberty, hyperkalemia, severe parkinsonism, acute respiratory alkalosis, tachypnea and abnormal prolactin levels are also reported after ETV. Frequent occurrence of diabetes insipidus and hypothalamic dysfunction in these patients warrants postoperative monitoring of serum electrolyte levels in all these patients [39]. There can be significant morbidity due to late infectious complications, such as meningitis and ventriculitis; so all patients should be monitored for signs of CNS infection [27,39].

\section{Conclusion}

Neuroendoscopy has advanced both as an independent treatment modality and as an adjunct to micro-neurosurgery for various neurologic disorders. Anaesthesiologists can positively contribute to overall neurologic outcome of these procedures through attention to the medical condition of the patient, the basic surgical requirements, specific anaesthetic requirements, and heightened awareness of specific intraoperative and postoperative risks. General anaesthesia with endotracheal intubation remains the technique of choice. Though this is a minimally invasive procedure, invasive haemodynamic monitoring, including direct or indirect measures of ICP are crucial to identify sudden and dangerous increases in ICP. Bradycardia is the commonest arrhythmia seen intraoperatively and generally respond to simple manoeuvres like removal of scope, decreasing the speed of inflow of irrigation fluid and allowing its egress. Use of atropine and other resuscitative measures are needed rarely. Warmed RL can be safely used for intraoperative irrigation with minimal postoperative impact. Close observation of vital signs, serum electrolytes as well as volume and temperature of the irrigation fluid and close communication between anesthesiologist and surgeon, are prerequisites for better outcome. Close postoperative monitoring is required to diagnose and treat complications such as convulsions, persistent hydrocephalus, blocked stoma, CSF leak, haemorrhage, post-operative intracranial hematomas, diabetes insipidus, electrolyte imbalance and infections thus improving overall outcome.

\section{References}

1. Enchev Y, Oi S (2008) Historical trends of neuroendoscopic surgical techniques in the treatment of hydrocephalus. Neurosurg Rev 31(30): 249-262.

2. Ersahin Y, Arslan D (2008) Complications of endoscopic third ventriculostomy. Childs Nerv Syst 24(8): 943-948.

3. Baykan N, Isbir 0, Gercek A (2005) Ten years of experience with paediatric neuroendoscopic third ventriculostomy: Features and perioperative complications of 210 cases. J Neurosurg Anaesthesiol 17(1): 33-37.

4. Dandy WE (1922) an operative procedure for hydrocephalus. Johns Hopkins Hosp Bull 33: 189-190. 


\section{Anaesthesia \& Critical Care Medicine Journal}

5. Dandy WE (1922) Cerebral ventriculoscopy. Johns Hopkins Hosp Bull 33: 181.

6. Mixter WJ (1923) Ventriculoscopy and puncture of the floor of the third ventricle: Preliminary report of a case. Boston Med Surg J 188: 277-278.

7. Oi S, Hidaka M, Honda Y, Togo K, Shinoda M, et al. (1999) Neuroendoscopic surgery for specific forms of hydrocephalus. Childs Nerv Syst 15(1): 56-68.

8. Paladino J, Rotim K, Heinrich Z (1998) Neuroendoscopic fenestration of arachnoid cysts. Minim Invasive Neurosurg 41(3): 137-140.

9. Gaab MR, Schroeder HW (1998) Neuroendoscopic approach to intraventricular lesions. J Neurosurg 88(3): 496-505.

10. Jones RFC, Stening WA, Brydon M (1990) Endoscopic third ventriculostomy. Neurosurgery 26(1): 86-92.

11. Cinalli G, Salazar C, Mallucci C, Yada JZ, Zerah M, et al. (1998) The role of endoscopic third ventriculostomy in the management of shunt malfunction. Neurosurgery 43(6): 1327-1329.

12. Hopf NJ, Grunert P, Fries G (1999) Endoscopic third ventriculostomy: Outcome analysis of 100 consecutive procedures. Neurosurgery 44(4): 795804.

13. Brockmeyer D, Abtin K, Carey L, Walker ML (1998) Endoscopic third ventriculostomy: An outcome analysis. Pediatr Neurosurg 28(5): 236-240.

14. Mohanty A, Das BS, Kolluri VRS, Hedge T (1996) Neuro-endoscopic fenestration of occluded foramen of Monro causing unilateral hydrocephalus. Pediatr Neurosurg 25(5): 248-251.

15. Ruge JR, Johnson RF, Bauer J (1996) Burr hole neuroendoscopic fenestration of quadrigeminal cistern arachnoid cyst: Technical case report. Neurosurg 38(4): 830-837.

16. Zymberg ST (2013) Neurocysticercosis. World Neurosurg 79(2): 24-28.

17. Griffith HB, Jamjoom AB (1990) The treatment of childhood hydrocephalus by choroid plexus coagulation and artificial cerebrospinal fluid perfusion. Br J Neurosurg 4(2): 95-100.
18. Bauer BL, Hellwig D (1994) Minimally invasive endoscopic neurosurgery-A survey. Acta Neurochir Suppl (Wien) 61: 1-12.

19. Alvarez JA, Cohen AR (1998) Neonatal applications of neuroendoscopy. Neurosurg Clin N Am 9(2): 405-413.

20. Margetis K, Souweidane MM (2013) Endoscopic treatment of intraventricular cystic tumors. World Neurosurg 79(2): 19-21.

21. Fabregas N, Valero R, Carrero E, Tercero J, Caral L, et al. (2001) Episodic high irrigation pressure during surgical neuroendoscopy may cause intermittent intracranial circulatory insufficiency. J Neurosurg Anesthesiol 13(2): 152-157.

22. Zwass MS, Fisher DM, Welborn LG, Cote CJ, Davis PJ, et al. (1992) Induction and mainte- nance characteristics of anaesthesia with desflurane and nitrous oxide in infants and children. Anesthesiology 76(3): 373-378.

23. Olsson GL (1995) Inhalational anaesthesia at the extremes of age: paediatric anaesthesia. Anaesthesia 50: 34-36.

24. Derbent A, Ersahin Y, Yurtseven T, Turhan T (2006) Hemodynamic and electrolyte changes in patients undergoing neuroendoscopic procedures. Childs Nerv Syst 22(3): 253-257.

25. Van Aken J, Struys M, Verplancke T, de Baerdemaeker L, Caemaert J, et al. (2003) Cardiovascular changes during endoscopic third ventriculostomy. Minim Invasive Neurosurg 46(4): 198-201.

26. Kalmar AF, Van AJ, Caemaert J, Mortier EP, Struys MM (2005) Value of Cushing reflex as warning sign for brain ischaemia during neuroendoscopy. Br J Anaesth 94: 791-799.

27. Ambesh SP, Kumar R (2000) Neuroendoscopic procedures: anesthetic considerations for a growing trend: A review. J Neurosurg Anesthesiol 12(3): 262270.

28. Ganjoo P, Sethi S, Tandon MS, Singh D, Pandey BC (2010) Perioperative complications of intraventricular neuroendoscopy: a 7-year experience. Turk Neurosurg 20(1): 33-38.

29. Salvador L, Valero R, Carrero E, Caral L, Fernandez S, et al. (2007) Cerebrospinal fluid composition 


\section{Anaesthesia \& Critical Care Medicine Journal}

modification after neuroendoscopic procedures. Minim Invasive Neurosurg 50(1): 51-55.

30. El Dawlatly AA (2004) Blood biochemistry following endoscopic third ventriculostomy. Minim Invasive Neurosurg 47(1): 47-48.

31. Kalmar AF, Van AJ, Struys MM (2005) Exceptional clinical observation: total brain ischemia during normal intracranial pressure readings caused by obstruction of the outflow of a neuroendoscope. J Neurosurg Anesthesiol 17(3): 175-176.

32. Anandh B, Madhusudan Reddy KR, Mohanty A, Umamaheswara Rao GS, Chandramouli BA (2002) Intraoperative bradycardia and postoperative hyperkalemia in patients undergoing endoscopic third ventriculostomy. Minim Invasive Neurosurg 45(3): 154-157.

33. Cinalli G, Spennato P, Ruggiero C, Aliberti F, Zerah M, et al. (2006) Intracranial pressure monitoring and lumbar puncture after endoscopic third ventriculostomy in children. Neuro- surgery 58(1): 126-136.

34. Jimenez-Vazquez OH, Nagore $\mathrm{N}$ (2008) The impact of neuroendoscopy in the emergency setting: a retrospective study of imaging, intraoperative findings, and surgical outcome in 55 patients. Clin Neurol Neurosurg 110(6): 539-543.

35. Kalmar AF, De Ley G, Van Den Broecke C, Van Aken J, Struys MM, et al. (2009) Influence of an increased intracranial pressure on cerebral and systemic haemodynamics during endoscopic neurosurgery: an animal model. Br J Anaesth 102(3): 361-368.

36. Fabregas N, Lopez A, Valero R, Carrero E, Caral L, et al. (2000) Anesthetic management of surgical neuroendoscopies: Usefulness of monitoring the pressure inside the neuroendoscope. J Neurosurg Anesthesiol 12(1): 21-28.

37. Salvador L, Valero R, Carazo J, Caral L, Rios J, et al. (2010) Pressure inside the neuroendoscope: correlation with epidural intracranial pressure during neuroendoscopic procedures. J Neurosurg Anesthesiol 22(3): 240-246.

38. Fabregas N, Craen RA (2006) Pressure inside the neuroendoscope should be measured on the inflow channel. J Neurosurg Anesthesiol 18(2): 161- 163.
39. Schroeder HW, Niendorf WR, Gaab MR (2002) Complications of endoscopic third ventriculostomy. J Neurosurg 96(6): 1032-1040.

40. Alfery D, Shapiro H, Gagnon R (1980) Cardiac arrest following rapid drainage of cerebrospinal fluid in a patient with hydrocephalus. Anesthesiology 52(5): 443-444.

41. Handler MH, Abbott R, Lee M (1994) A near-fatal complication of endoscopic third ventriculostomy: Case report. Neurosurgery 35(3): 525-527.

42. El-Dawlatly AA, Murshid WR, Elshimy A, Magboul MA, Samarkandi A, et al. (2000) The incidence of bradycardia during endoscopic third ventriculostomy. Anesth Analg 91(5): 1142-1144.

43. Proano JV, Torres-Corzo J, Rodriguez-Della VR, Guizar-Sahagun G, Rangel-Castilla L (2009) Intraventricular and subarachnoid basal cisterns neurocysticercosis: a comparative study between traditional treatment versus neuroendoscopic surgery. Childs Nerv Syst 25(11): 1467-1475.

44. Ganjoo P, Sethi S, Tandon MS (2009) Incidence and pattern of intraoperative hemodynamic response to endoscopic third ventriculostomy. Neurol India 57(2): 162-165.

45. Yadav YR, Parihar V, Kher Y (2013) Complication avoidance and its management in endoscopic neurosurgery. Neurol India 61(3): 217-225.

46. Singh GP, Prabhakar H, Bithal PK, Dash HH (2011) A retrospective analysis of perioperative complications during intracranial neuroendoscopic procedures: Our institutional experience. Neurol India 59(6): 874-878.

47. Yadav YR, Parihar V, Pande S, Namdev H, Agarwal M (2012) Endoscopic third ventriculostomy. J Neurosci Rural Pract 3(2): 163-173.

48. El Sawaf Y, Shafik I, Baza R, Torky S (2010) Complications and failures of endoscopic third ventriculostomy: Perception of their avoidance. Pan Arab J Neurosurg 14: 69-74.

49. Salvador L, Hurtado P, Valero R, Tercero J, Carrero E, et al. (2009) Importance of monitoring neuroendoscopic intracranial pressure during anesthesia for neuroendoscopic surgery: review of 101 cases. Rev Esp Anestesiol Reanim 56(2): 75-82. 


\section{Anaesthesia \& Critical Care Medicine Journal}

50. Enya S, Masuda Y, Terui K (1997) Respiratory arrest after a ventriculoscopic surgery in infants: Two case reports, Masui 46(3): 416-420. 